\title{
Dynamique de l'abondance des oocystes d'Isospora belli dans un milieu aquatique en zone tropicale (Cameroun)
}

\section{Abundance dynamics of the oocystic load of Isospora belli in a tropical aquatic medium (Cameroon)}

\author{
Ajeagah Gideon*, Karie Mouncharou Jean E.
}

\author{
Laboratoire d'Hydrobiologie et Environnement, Université de Yaoundé I, Faculté des Sciences, BP 812, \\ Yaoundé, Cameroun \\ ajeagahg@yahoo.com
}

\begin{abstract}
Résumé - Une étude visant à isoler, identifier et caractériser les oocystes d'l. belli dans l'Abiergué a été menée d'avril à novembre 2011. Les échantillonnages ont été faits mensuellement dans six stations le long du cours d'eau. Les oocystes ont été identifiés par des techniques de coloration notamment la coloration de Zielh-Neelsen modifiée et celle du Lugol. Les résultats de la biologie révèlent la présence et la distribution des oocystes $d$ 'l.belli le long du cours d'eau avec une densité moyenne de 344 oocystes/L. Cette abondance résulte de la contamination par les matières fécales déversées dans l'hydrosystème à partir des latrines-canons. Les oocystes identifiés montrent une prédominance des oocystes à sporoblaste non-individualisé, en moyenne 202 oocystes/L, aussi bien au niveau spatial que saisonnier. Ce travail a mis en évidence la dominance des oocystes de petite taille avec une densité moyenne de 194 oocystes/L dans ce milieu aquatique en zone urbaine. Le calcul du coefficient de corrélation de Spearman montre des corrélations positivement significatives $(p=0,01)$ entre les nitrates, la turbidité et les oocystes d'une part et une corrélation négativement significative $(p=0,05)$ entre le $\mathrm{CO}_{2}$ dissous et les oocystes d'autre part.
\end{abstract}

Mots-clés - abondance, Abiergué, protozoaire, Isospora belli, oocystes

\begin{abstract}
A study which consists of isolating, identifying and characterizing the oocysts of I. belli, was carried out from April to November 2011 in the Abiergue Stream. The sampling was done monthly on six sites along the stream. The oocysts were identified by staining techniques namely modified Zielh-Neelsen and Lugol stains. The physico-chemical analyses reveal that Abiergué sustains an organic pollution pressure. The results of biology show the presence and the distribution of $I$. belli oocysts along Abiergué with an average density of 344 oocysts/L. This value of abundance arises from the contamination of the stream by fecal matters which are released from the latrines. Identified oocysts show a predominance of non-individualized sporoblast oocysts with an average density of 202 oocysts/L in all the seasons and in all the sampling sites. This research has revealed the dominance of small
\end{abstract}


size oocysts with an average density of 194 oocysts/L in this urban water system. The calculation of Spearman correlation coefficient shows positive significant correlations $(p=0,01)$ between nitrates, turbidity and oocysts. However, the dissolved $\mathrm{CO}_{2}$ has a negative significant correlation with the oocysts.

Key words - Abiergué, abundance, protozoa, Isospora belli, oocysts

\section{INTRODUCTION}

L'eau est une ressource naturelle vitale et importante pour toutes les composantes de divers écosystèmes du globe. L'avoir en quantité suffisante et de bonne qualité rentre dans les objectifs du millénaire pour le développement au regard de la vitesse croissante de dégradation de cette ressource (ONU, 2006). Toutefois, l'activité anthropique figure au premier rang des problèmes responsables de la pollution de l'eau douce à l'échelle mondiale. Cette situation exige une gestion rigoureuse et un suivi permanent des hydrosystèmes qui, sous l'action de l'anthropisation, constituent le siège de la prolifération et de la dissémination de divers agents pathogènes tels que les virus, les champignons, les bactéries et les protozoaires parasites (Gomez et al., 2002). En effet, dans les pays en développement, plus d'un milliard de personnes n'ont pas accès à l'eau potable et trois milliards de personnes environ n'ont pas accès aux services d'assainissement adéquats avec une majorité vivant en Afrique et en Asie (Sonia et al., 2010). Ces insuffisances accentuées par les perturbations climatiques, l'industrialisation et l'explosion démographique favorisent la contamination des eaux par les fèces et divers effluents domestiques et industriels, la dégradation croissante et l'épuisement des ressources en eau (Pedro \& Germano, 2001 ; Mircean et al., 2012).

Au Cameroun en général et dans la ville de Yaoundé en particulier, les populations sont confrontées au problème d'assainissement et aux difficultés d'approvisionnement en eau potable. Pour pallier ce problème, elles ont recours à des sources, des puits et des cours d'eau pour satisfaire leur besoin. Dans cette quête, elles s'exposent à des parasitoses d'origine hydrique et de nature diarrhéique dont la prévalence est autour de $14,4 \%$ (Yongsi et al., 2008). Parmi les parasitoses, figure l'isosporose, causée par Isospora belli qui est responsable des maladies diarrhéiques chez les immuno-compétents et surtout les immuno-déprimés (VIH/SIDA) (Sasaki et al., 2004 ; Navaneethan et al., 2012). Cette entéropathologie est d'une gravité particulière chez les porteurs du VIH en raison d'une expression clinique sévère et superposable à celle de la cryptosporidiose (Cranenbonk et al., 2003) car ces deux pathologies présentent presque les mêmes symptômes. I. belli, Cryptosporidium et Cyclospora sont des Apicomplexa à émergence qui se retrouvent dans les infections concomitamment d'où des co-infections dues à la nature de leur forme infestante qui est le sporozoïte. Le traitement de référence pour l'isosporose est la prise orale de l'association 
Trimethoprime-sulfamethoxazole (160 ou $800 \mathrm{mg}$ ) ou le Ciplofloxacine $(500 \mathrm{mg}$ ) deux fois par jour pendant sept jours chez les immunodéprimés (Verdier et al., 2000).

Les travaux d'Ajeagah et al. (2007) ont montré la présence des formes de résistance des protozoaires du genre Giardia et Cryptosporidium dans le présent cours d'eau ainsi que ceux de Kenmogne et al. (2010) qui ont montré la dissémination des entéropathogènes comme des coliformes fécaux et totaux, des œufs d'Helminthes et des kystes d'Entamoeba hystolitica dans l'Abiergué. Au regard de quelques similitudes autour des symptômes de gastroentérites causées d'une part et de leur cycle de développement d'autre part, il était intéressant pour nous d'observer si les oocystes de l. belli ne se retrouvaient pas aussi dans le cours d'eau. Le cycle biologique de cet entéropathogène se subdivise en trois grandes phases dont la multiplication asexuée, la reproduction sexuée et la sporogonie (Fig. 1) : (i) la multiplication asexuée (dans les entérocytes humains) qui conduit à la libération de 8 sporozoïtes à partir d'un oocyste mature ingéré par l'homme, leur division en mérozoïtes et leur différenciation en gamétocytes et en gamètes ; (ii) la reproduction sexuée qui intervient une semaine après l'infection et consiste en une fertilisation du macrogamète par un microgamète issu des gamétocytes conduisant à la formation d'un oocyste ovale, immature (renfermant un sporoblaste), dont la taille varie de 20 à $33 \mu \mathrm{m}$, qui est rejeté avec la matière fécale dans le milieu extérieur; (iii) la sporogonie qui intervient dans les conditions favorables de l'environnement et correspond à la phase de maturation du sporoblaste. Ce sporoblaste subit une mitose aboutissant à 2 sporocystes lesquels subissent une méiose produisant 4 sporozoïtes (formes infestantes) chacun : c'est l'oocyste mature (Fig. 1).

À l'état actuel, nous ne disposons d'aucune information sur la distribution des coccidies entéropathogènes dans les hydrosystèmes à Yaoundé en dehors des travaux d'Ajeagah et al. (2007, 2010) et Kenmogne et al. (2010). Par ailleurs, les travaux sur I. belli sont essentiellement menés par examen direct des échantillons de la matière fécale en laboratoire comme ceux de Udeh et al. (2008) et Meamar et al. (2009) et non à partir des échantillons d'eau des cours d'eau. À cet effet, on procède par prélèvement de selles ou aspiration duodénale suivie par des techniques de coloration comme celle de Ziehl-Neelsen modifiée (Ousmane, 2010) ainsi que celle au Lugol.

Compte tenu du fait que $I$. belli a besoin d'un passage hors de l'hôte pour assurer sa maturité et que, de plus, les oocystes conservent leur pouvoir infectant pendant de longues périodes dans l'eau, la présente étude vise à isoler, identifier et caractériser les oocystes d'l. belli dans un cours d'eau, l'Abiergué.

\section{MATÉRIEL ET MÉTHODES}

\subsection{Site d'étude et échantillonnage}

Yaoundé, capitale politique du Cameroun, est situé sur la bordure 
maturation de l'oocyste (environ 2 semaines) dans le milieu extérieur

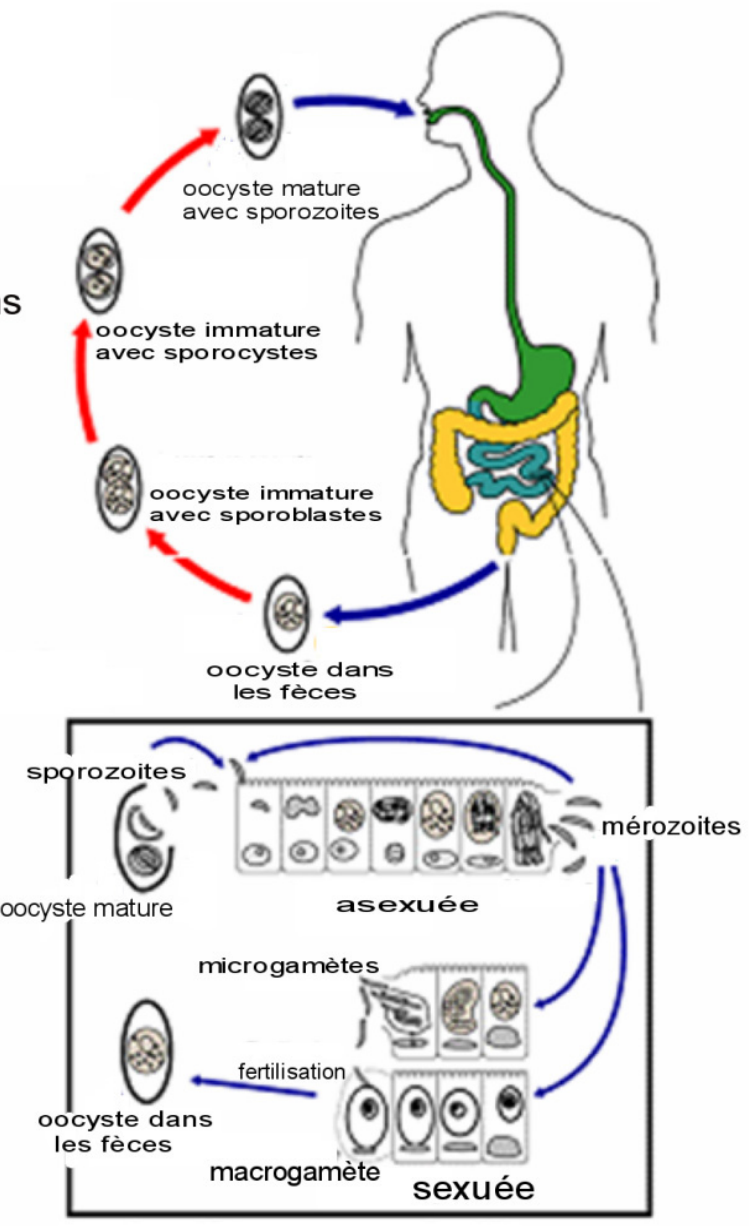

Fig. 1. Cycle de développement d'Isospora belli (CDC, 2007).

Fig. 1. Life cycle of Isospora belli (CDC, 2007).

ouest du plateau sud Camerounais à $3^{\circ} 52^{\prime}$ de latitude Nord et $11^{\circ} 32$ ' de longitude Est, et culminant à une altitude moyenne d'environ $750 \mathrm{~m}$ (Santoir, 1995). Le relief est accidenté et étendu sur plusieurs collines d'altitude comprises entre 25 et 50 mètres, les sols sont dans la globalité de type ferrolatéritique (Yongué-Fouateu, 1986). Le substratum géologique est constitué d'un socle granito-gneissique sur lequel se développent des sols ferralitiques et hydromorphes (Ndam Ngoupayou et al., 2007).

Le climat est équatorial de type yaoundéen chaud et humide, caractérisé par des précipitations modérées et une température variable au cours du temps (Suchel, 1987). Ces caractéristiques permettent de distinguer 
4 saisons : la grande saison sèche (GSS) de mi-novembre à mi-mars, une petite saison pluvieuse (PSP) de mi-mars à fin juin, la petite saison sèche (PSS) de juillet à mi-août et une grande saison pluvieuse (GSP) de mi-août à mi-novembre.

L'Abiergué est l'un des affluents du cours d'eau Mfoundi. Son débit est fonction du climat, du relief du bassin versant, de la variation de saisons, de la stabilité de fonds et des apports des tributaires (Levêque, 2001). Le débit varie de $0,1 \pm 0,04 \mathrm{~m}^{3} / \mathrm{s}$ à la source, $0,5 \pm 0,1 \mathrm{~m}^{3} / \mathrm{s}$ au milieu et $0,7 \pm$ $0,2 \mathrm{~m}^{3} / \mathrm{s}$ en aval selon les variables hydro-météologiques. II reçoit en dehors de ses deux affluents, plusieurs effluents provenant des eaux usées domestiques et des commerces. Son bassin versant est essentiellement occupé par des lotissements, les marchés et une agriculture maraîchère artisanale avec l'emploi des intrants agricoles. Les activités pratiquées sont principalement commerciales (les marchés $\mathrm{VIII}{ }^{\mathrm{e}}$ et Mokolo), et agricoles avec quelques champs de légumes le long du cours d'eau à des fins de subsistance et utilisant l'eau du cours d'eau pour arroser les plantes. D'une manière générale, la densité de la population est très forte avec des maisons en matériau provisoire et dépourvues de dispositif d'assainissement.

Ainsi, on peut observer de nombreuses latrines bâties sur les berges, qui déversent directement leur contenu dans le cours d'eau (en moyenne tous les 20 mètres). De plus, des tas d'ordures sont présents de part et d'autre du cours d'eau et même dans le lit, par endroit, rendant difficile l'écoulement des eaux. Cependant, les pressions anthropiques à travers les pollutions fécales et les déchets domestiques ne sont pas distribuées de façon uniforme tout au long du cours d'eau puisque les activités diffèrent.

Sur la base de la densité des populations des quartiers riverains $\mathrm{du}$ l'hydrosystème, du degré d'insalubrité, des effluents domestiques et des affluents ainsi que des sources de pollution, six stations ont été choisies dans l'Abiergué, trois au crénon, deux au rithron et un au potamon (Fig. 2). Les échantillonnages ont été menés sur l'Abiergué pendant la période allant d'avril à novembre 2011, suivant une fréquence de prélèvement mensuel et les résultats ramenés aux saisons à partir des moyennes des valeurs mensuelles des différents paramètres. Ainsi, pendant la PSP, il y a eu trois échantillonnages contre deux en PSS et enfin trois au cours de la GSP. Les spécimens ont été prélevés dans des flacons en polyéthylène de 1 litre et les échantillons ont ensuite été transportés à température ambiante dans une glacière au Laboratoire de biologie générale de l'université de Yaoundé I dans environ l'heure qui suit le prélèvement pour analyse.

\subsection{Analyses physico-chimiques}

Les prélèvements ainsi que les paramètres utilisés ont été effectués de manière ponctuelle entre $7 \mathrm{H} 30$ et $11 \mathrm{H} 30$ du matin sur le terrain. La température qui varie très peu en zone tropicale, a été mesurée à l'aide d'un thermomètre à mercure, le $\mathrm{pH}$ grâce au $\mathrm{pH}$-mètre $(\mathrm{HACH})$ et $\mathrm{l}^{\prime} \mathrm{O}_{2}$ dissous à l'aide de l'oxymètre $(\mathrm{HACH})$ in situ. 


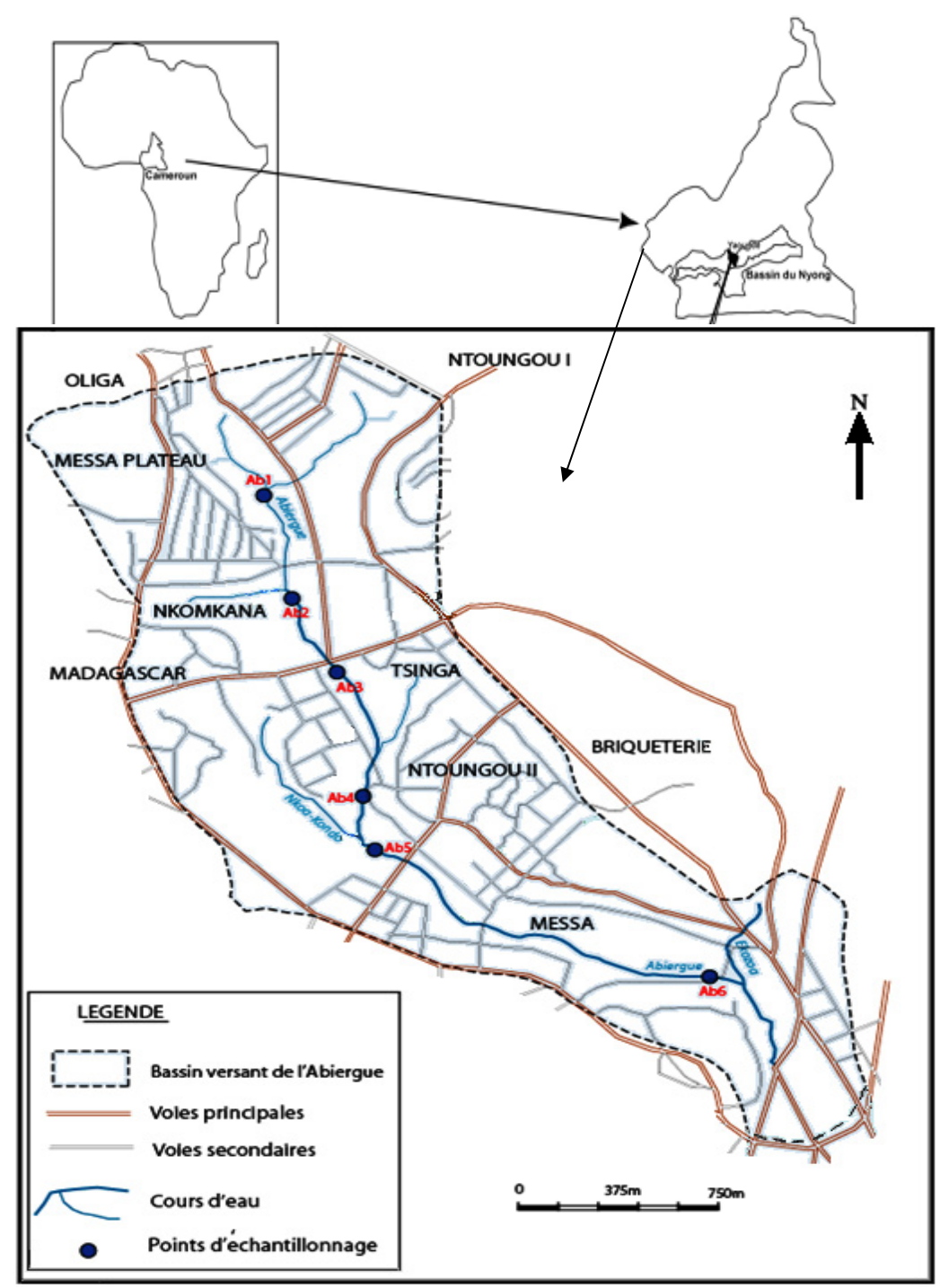

Fig. 2. Carte du bassin versant de l'Abiergué comprenant les stations d'échantillonnage (Source: Carte topographique au 1/10000 de l'INC, Yaoundé - Cameroun 2008, modifié).

Fig. 2. Map of the Abiergue drainage basin indicating the sampling stations (Source: Topographic map of 1/10000 of INC, Yaoundé - Cameroon 2008, modified).

Ces trois paramètres sont importants dans le processus de sporogonie par l'activation des enzymes, le métabolisme et le phénomène d'excystation.
La turbidité qui est mesurée par spectrophotomètre DR2800 permet de comprendre le déplacement des oocystes-matières en suspension dans 
les milieux aquatiques. Les nitrates ont été mesurés par colorimétrie au spectrophotomètre (DR2800) au laboratoire. Le dioxyde de carbone $\left(\mathrm{CO}_{2}\right)$ a été évalué par volumétrie au laboratoire après fixation au $\mathrm{NaOH} \mathrm{N} / 20$ sur le terrain. L'oxydabilité au permanganate déterminant la quantité de constituants organiques oxydables a été évaluée au laboratoire par volumétrie. Les nitrates $\left(\mathrm{mg} \mathrm{NO}_{3} / \mathrm{L}\right)$ et le $\mathrm{CO}_{2}$ dissous ( $\mathrm{mg} \mathrm{CO}_{2} / \mathrm{L}$ ) sont des indicateurs de la pollution anthropogénique (Ajeagah et al., 2013). Les nitrates retrouvés dans les cours d'eau proviennent régulièrement du lessivage des sols agricoles (où se pratiquent les cultures maraîchères) et des processus bactériens du cycle de l'azote à partir des matières organiques (Rodier, 2009). Le $\mathrm{CO}_{2}$ provient de la pluie, la respiration des organismes et la décomposition de matières organiques (Ajeagah et al., 2013). Ces paramètres ont été choisis par rapport à l'écologie d'l. belli en milieux naturels et sur la base des paramètres utilisés dans des travaux antérieurs sur les protozoaires du genre Giardia et Cryptosporidium par Ajeagah et al. (2007, 2010).

\subsection{Analyses biologiques}

En ce qui concerne les oocystes d'l. belli, les échantillons d'eau prélevés dans les flacons en polyéthylène (1 litre) ont été par la suite laissés sédimenter pendant 24 à 48 heures à température ambiante autour de $27^{\circ} \mathrm{C}$ en moyenne. Cet intervalle de temps est idéal pour le dépôt de la majorité des oocystes comme présenté par Ajeagah et al. (2007). Après les 48 heures, les oocystes peuvent être ingérés par des bactéries et d'autres prédateurs présents dans l'échantillon. Les culots obtenus ont été homogénéisés et $20 \mathrm{~mL}$ prélevés pour chaque échantillon ont été repartis dans 4 tubes à essai. Compte tenu des objectifs d'observation, deux méthodes ont été utilisées : (a) la méthode d'observation directe car le travail consiste en une évaluation de l'abondance et le comptage des oocystes est aisé pour une évaluation quantitative ; (b) la méthode d'observation après coloration de ZielhNeelsen modifié car cette méthode est plus qualitative et permet la confirmation de la présence des oocystes.

Pour l'observation directe, les contenus des tubes ont été fixés au formaldehyde $2 \%$, puis colorés au Lugol $5 \%$ et portés à centrifugation pendant 10 minutes à 500 trs/min. Après la centrifugation, le culot a été prélevé à l'aide d'une micropipette, déposé sur une lame et recouvert d'une lamelle et observé au microscope optique (Olympus) aux grandissements $40 \mathrm{X}$ et $100 \mathrm{X}$ pour identification, mensuration et dénombrement des oocystes jusqu'à épuisement du culot.

Par contre, pour l'observation après coloration de Zielh-Neelsen modifié, une solution de sulfate de zinc $10 \%$ est ajoutée au contenu des tubes pour favoriser la flottaison, après fixation au formaldehyde $2 \%$, puis l'ensemble est centrifugé à $500 \mathrm{trs} / \mathrm{min}$ pendant 10 minutes. Le surnageant est prélevé à l'aide d'une micropipette et posée sur des lames porte-objet. Après fixation au méthanol et coloration à la fuchsine basique, on rince à l'eau puis à l'acide sulfurique $2 \%$. On réalise ensuite une contre-coloration au bleu de méthylène 
Tableau I. Coefficients de corrélation obtenus entre quelques variables physico-chimiques mesurées, la taille des oocystes et les différents stades de sporulation.

Table I. Correlation coefficients between the evaluated physico-chemical variables, the size of the oocysts and the different stages of sporulation.

\begin{tabular}{|l|c|c|c|c|c|c|c|}
\hline & Température & $\begin{array}{c}\text { Oxygène } \\
\text { dissous }\end{array}$ & Turbidité & Nitrates & $\begin{array}{c}\mathrm{CO}_{2} \\
\text { dissous }\end{array}$ & $\mathrm{pH}$ & Oydabi-lité \\
\hline $\begin{array}{l}\text { Sporoblaste } \\
\text { immature }\end{array}$ & 0,378 & $-0,426$ & 0,418 & 0,402 & $-0,404$ & $-0,663^{\star \star}$ & $-0,364$ \\
\hline $\begin{array}{l}\text { Sporoblaste } \\
\text { mature }\end{array}$ & $-0,198$ & $-0,459$ & $-0,076$ & 0,304 & $-0,507^{*}$ & 0,097 & $-0,022$ \\
\hline Sporocystes & $-0,365$ & $-0,38$ & $-0,198$ & $0,566^{\star}$ & $-0,518^{\star}$ & 0,193 & 0,024 \\
\hline Petite taille & $-0,24$ & $-0,631^{\star *}$ & $-0,08$ & 0,293 & $-0,43$ & 0,006 & $-0,026$ \\
\hline $\begin{array}{l}\text { Taille } \\
\text { moyenne }\end{array}$ & 0,008 & $-0,582^{\star}$ & 0,115 & $0,516^{\star}$ & $-0,629^{\star *}$ & $-0,213$ & $-0,218$ \\
\hline Grande taille & $0,555^{\star}$ & $-0,492^{\star}$ & $0,680^{\star *}$ & $0,625^{\star \star}$ & $-0,157$ & $-0,671^{\star \star}$ & $-0,520^{\star}$ \\
\hline
\end{tabular}

* La corrélation est significative au niveau 0,05.

${ }^{\star *}$ La corrélation est significative au niveau 0,01 .

$5 \%$ puis, après rinçage à l'eau et séchage à l'air, l'examen des oocystes est réalisé au microscope optique (Olympus) aux grandissements 40X et 100X.

\subsection{Analyses des données}

Après l'enregistrement du volume intégral du culot $\left(V_{X}\right)$ et son homogénéisation, un volume précis du culot $\left(V_{Y}\right)$ est prélevé puis reparti dans différents tubes à essai. Le culot final de chaque tube à essai est reparti sur les lames puis le nombre d'oocystes est compté. Le nombre total des oocystes dans l'échantillon est calculé en multipliant la valeur obtenue pour toutes les lames par la fraction $V_{X} / V_{Y}$ et le résultat est enfin ramené au litre. Les relations entre la distribution des oocystes du parasite et les variables physicochimiques de l'eau ont été évaluées par le test de corrélation de Spearman (Tab. I).

\section{RÉSULTATS ET DISCUSSION}

\subsection{Caractéristiques physico-chimiques}

\subsubsection{Paramètres physiques}

La température des eaux est comprise entre 22,40 et $26,53{ }^{\circ} \mathrm{C}$ dans l'ensemble du réseau avec une amplitude thermique entre la valeur minimale et maximale de $4,13^{\circ} \mathrm{C}$. La station Abiergué $2\left(A_{2}\right)$ au crénon pendant la PSS enregistre la plus faible valeur contrairement à la station Abiergué $6\left(\mathrm{Ab}_{6}\right)$ au potamon qui présente la valeur la plus élevée pendant la PSP (Fig. 3A). La variabilité de la température résulte selon Villeneuve et al. 


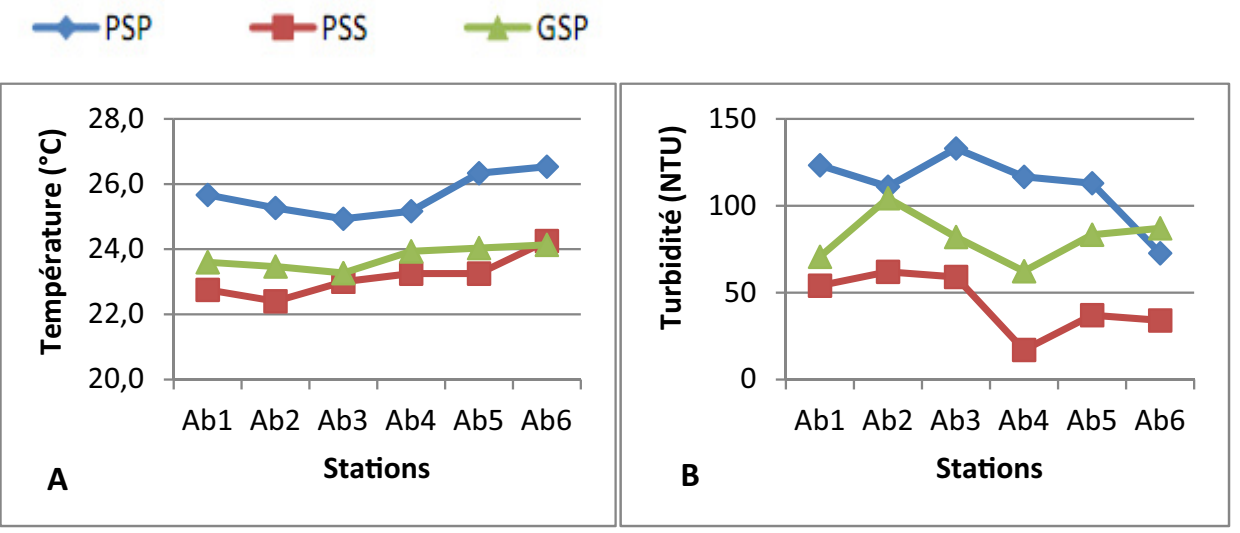

Fig. 3. Variation spatio-temporelle de la température (A) et de la turbidité (B) pendant la période d'étude.

Fig. 3. Spatio-temporal variation of temperature (A) and turbidity (B) during the study period.

(2006) de l'étroitesse liaison entre la température de l'eau et celle du milieu ambiant ainsi que d'éventuels rejets d'eaux résiduaires comme c'est le cas avec les ménages riverains et les nettoyeurs de l'abattoir traditionnel des volailles du marché VIII ${ }^{\mathrm{e}}$ qui évacuent les eaux usées chaudes dans le cours d'eau.

La turbidité de l'eau est comprise entre 34 et 133 NTU. La valeur maximale est obtenue à $A b_{3}$ au crénon en PSP et la valeur minimale est enregistrée à $A b_{6}$ au potamon en PSS (Fig. 3B). Dans l'ensemble, le profil de variation de la turbidité montre que la PSP et la GSP présentent les valeurs élevées résultant des torrents et des eaux de ruissellement qui drainent les particules solides et les maintiennent en suspension, rendant ainsi l'eau turbide. La diminution de la turbidité d'amont en aval en PSP et PSS résulterait d'une part d'un processus de dilution suite à l'apport de l'affluent en $\mathrm{Ab}_{5}$ et d'autre part du fait que le cours d'eau traverse une zone marécageuse entre l'amont $A b_{4}$ et l'aval de $A b_{5}$ avec sa fonction épuratrice qui réduirait la turbidité.

\subsubsection{Paramètres chimiques}

Le $\mathrm{pH}$ présente des valeurs comprises entre 6,86 et 7,81 respectivement sur $A b_{5}$ au rithron (PSP) et $A b_{6}$ (PSS) (Fig. 4C). Le profil de données montre une tendance vers la neutralité, propice à l'expression de nombreux organismes (Angelier, 2000). Ces résultats sont semblables à ceux obtenus par Kenmogne et al. (2010) sur l'Abiergué. Cette tendance résulterait de la qualité des déchets libérés dans le cours d'eau et de la nature acide du sol. $\mathrm{Ce} \mathrm{pH}$ acide qui provient de la nature des roches sous-jacentes et des sols drainés ainsi que de l'oxydation de la matière organique dans les eaux devient légèrement neutre à basique pour les eaux influencées par 

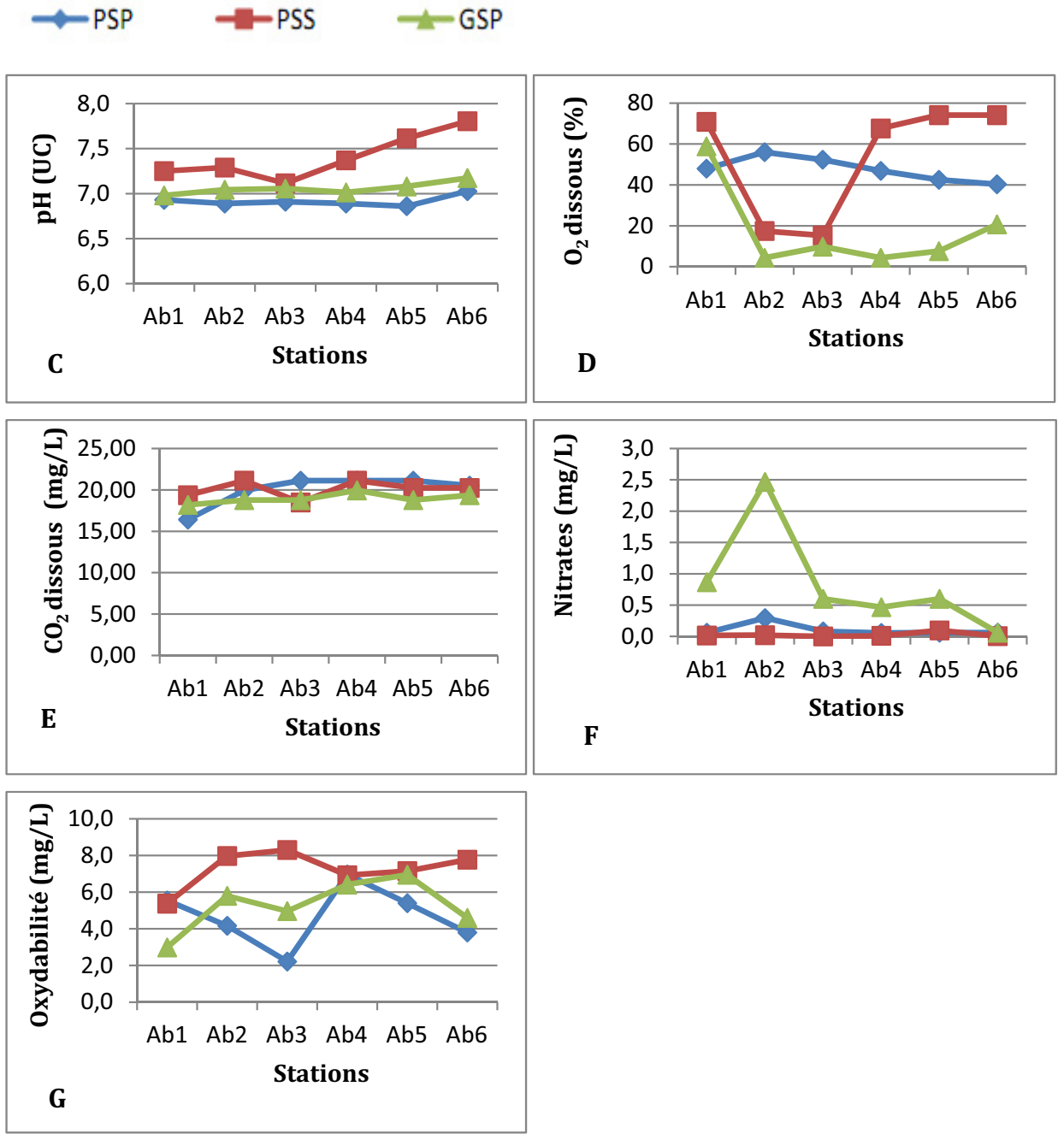

Fig. 4. Variation spatio-temporelle du $\mathrm{pH}(\mathrm{C})$, de l'O $\mathrm{O}_{2}$ dissous (D), du $\mathrm{CO}_{2}$ dissous (E), des nitrates (F) et de l'oxydabilité au permanganate $(\mathrm{G})$ pendant la période d'étude.

Fig. 4. Spatio-temporal variation of $\mathrm{pH}(\mathrm{C})$, dissolved $\mathrm{O}_{2}(\mathrm{D})$, dissolved $\mathrm{CO}_{2}(\mathrm{E})$, nitrates $(\mathrm{F})$ and oxydability $(\mathrm{G})$ during the study period.

les rejets urbains (Ndam Ngoupayou et al., 2007).

$\mathrm{L}^{\prime} \mathrm{O}_{2}$ dissous présente des valeurs comprises entre 4,36 et $74,12 \%$ (Fig. 4D). La teneur minimale est enregistrée à $A b_{2}$ au crénon et $A b_{4}$ au rithron pendant la GSP et la maximale à $A b_{5}$ et $A b_{6}$ (PSS).La forte teneur résulte de la pente et des radiers observés sur le lit au niveau des stations $A b_{1}$ et $A b_{6}$ favorables à la réoxygénation de l'eau à l'interface air/eau. 
Les teneurs en $\mathrm{CO}_{2}$ dissous varient entre $16,41 \mathrm{mg} / \mathrm{L}$ et $21,12 \mathrm{mg} / \mathrm{L}$. La valeur minimale est obtenue à $A b_{1}$ (PSP) et la valeur maximale à $A b_{3}, A b_{2}$ et $A b_{4}$ (PSP) et $A b_{2}$ et $A b_{4}$ (PSS) (Fig. 4E). Dans l'ensemble, le $\mathrm{CO}_{2}$ dissous présente peu de variations sur le plan spatio-temporel pendant la GSP, mais augmente de la source à l'exutoire pendant la PSP et la PSS.

Les teneurs en ions nitrates varient entre 0 et 2,47 $\mathrm{mgNO}_{3} / \mathrm{L}$ (Fig. 4F). De façon générale, la teneur reste inférieure à $0,5 \mathrm{mgNO}_{3} / \mathrm{L}$ en moyenne et $A b_{2}$ durant la GSP enregistre la teneur la plus élevée. La présence des nitrates dans le cours d'eau témoigne d'une contamination par les activités domestiques et maraîchères exercées sur le versant.

L'oxydabilité au permanganate présente des valeurs allant de 2,22 à $8,30 \mathrm{mgO}_{2} / \mathrm{L}$ (Fig. 4G). La plus faible teneur est observée à $A b_{3}$ (PSP) et la teneur la plus forte à $A b_{3}$ (PSS). Le profil de l'oxydabilité au permanganate présente dans son ensemble une teneur supérieure à $2 \mathrm{mgO}_{2} / \mathrm{L}$ que Rodier (2009) considère comme indicatrice de pollution dans le cas des eaux superficielles.

Le résultat d'analyse des paramètres physico-chimiques montre une dégradation de nature organique du ruisseau.

\subsection{Caractéristiques biologiques}

Les oocystes d'l. belli mesurent entre 20 à $30 \mu \mathrm{m}$ selon Garcia (2007) et Lindsay et al. (2007). Pour une harmonie dans le traitement des données au regard de l'étendue de la distribution des tailles au cours de l'étude, nous avons choisi délibérément de regrouper les oocystes en 3 gammes de taille: les oocystes de petite taille (20 à $23 \mu \mathrm{m})$, ceux de taille moyenne (24 à $26 \mu \mathrm{m}$ ) et ceux de grande taille (27 à $30 \mu \mathrm{m})$. Ce découpage permet de vérifier la fréquence des différentes types de taille dans notre environnement aquatique, de préconiser la nature de filtre à utiliser dans le cas de traitement des eaux et de commencer une analyse biologique sur le génotypage d'l. belli en zone équatoriale (sub-saharienne).

Dans l'ensemble, les analyses biologiques présentent la concentration maximale des oocystes à $A b_{1}$ (498 oocystes/L) en GSP et la concentration minimale à $A b_{5}$ (199 oocystes/L) en PSS. Les oocystes de petite taille présentent des abondances oscillant entre 117 et 296 oocystes/L (Fig. 5A). La plus faible abondance est relevée à $A b_{5}$ durant la PSS et la plus forte est observée à $A b_{1}$ durant la GSP. Les abondances des oocystes de taille moyenne varient entre 30 et 149 oocystes/L (Fig. 5B). L'abondance minimale est enregistrée à $A b_{4}$ en PSS et l'abondance maximale est obtenue à $\mathrm{Ab}_{4}$ en GSP. Les abondances des oocystes de grande taille varient entre 28 et 109 oocystes/L (Fig. 5C). La faible abondance est obtenue à $A b_{4}$ et $A b_{5}$ en PSS alors que la plus forte est relevée à $A b_{4}$ en GSP. Nous pouvons dire que les oocystes de petites tailles sont ceux que portent la majorité des hôtes car l'oocyste étant la forme de résistance, il ne peut pas changer de taille dans le milieu extérieur. La variabilité intra saisonnière des abondances des oocystes de petite et moyenne taille est plus importante contrairement aux abondances des oocystes de 

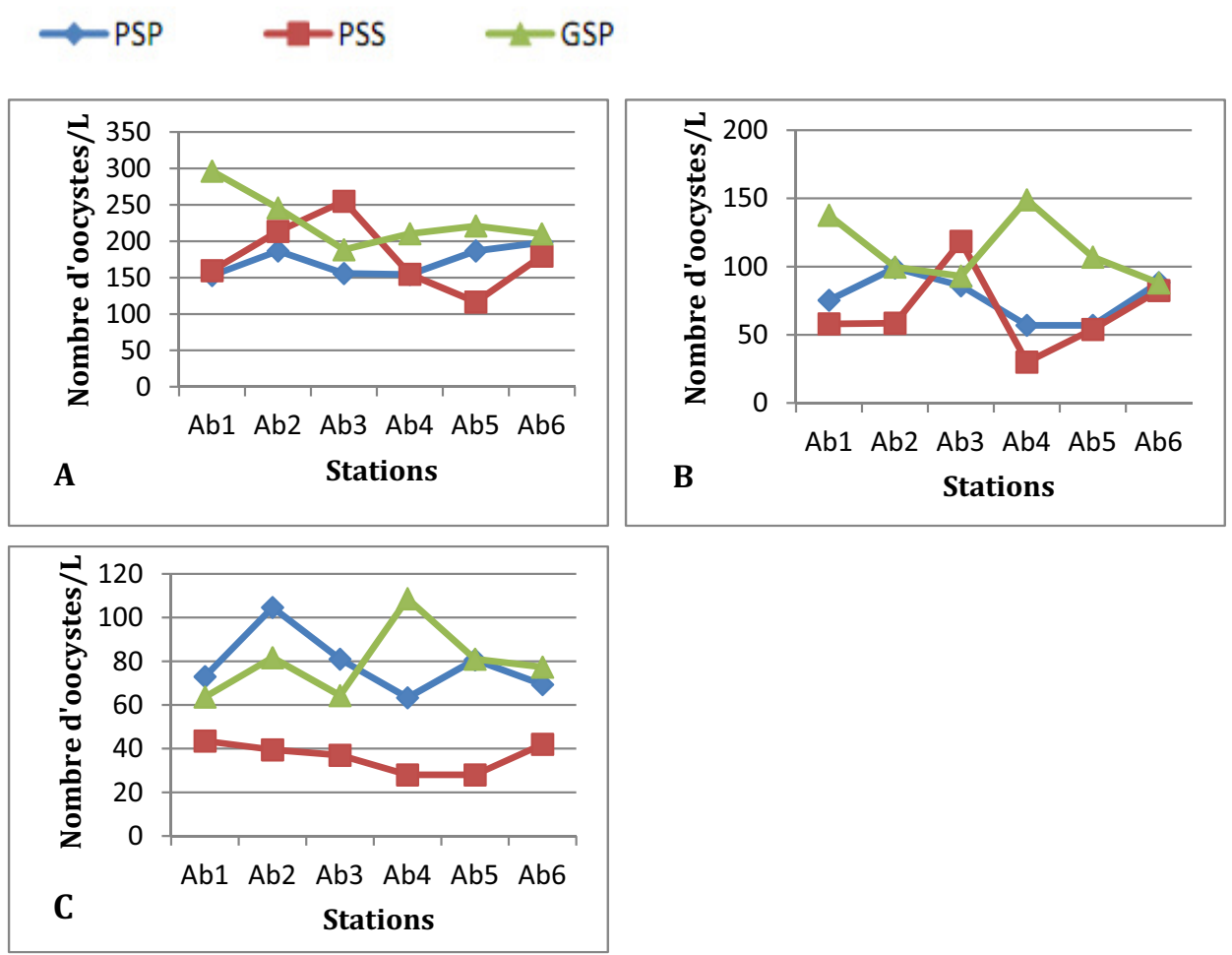

Fig. 5. Variation spatio-temporelle des abondances des oocystes de petite taille (A), de taille moyenne (B) et de grande taille (C) pendant la période d'étude.

Fig. 5. Spatio-temporal variation of small size oocysts, average height and large size during the study period.

grande taille qui présentent plutôt une variabilité inter saisonnière importante.

Par ailleurs, deux formes d'oocystes ont été identifiées : les non-sporulées (ceux à sporoblaste nonindividualisé (Fig. 6A) et ceux à un sporoblaste (Figs. 6B et 7A) et les sporulées (ceux à sporocystes) (Figs. $6 \mathrm{C}$ et $7 \mathrm{~B}$ ).

Les oocystes à sporoblaste nonindividualisés ou inexistant montrent des abondances variant entre 106 et 260 oocystes/L (Fig. 8A). Le profil des abondances révèle que la station $A b_{5}$ en PSS enregistre la plus faible valeur contrairement à la station $A b_{2}$ en PSP qui a la plus forte valeur. Aussi, les abondances des oocystes à un sporoblaste individualisé sont comprises entre 51 et 157 oocystes/L (Fig. 8B). La station $\mathrm{Ab}_{5}$ en PSS enregistre l'abondance minimale et la station $A b_{1}$ en GSP présente l'abondance maximale. Les abondances des oocystes à sporocystes fluctuent entre 20 et 99 oocystes/L (Fig. 8C). La station $A b_{1}$ en PSP obtient la plus faible abondance alors que la station $\mathrm{Ab}_{4}$ en GSP a l'abondance la plus élevée. La variabilité intra saisonnière des abondances des oocystes à sporoblaste 

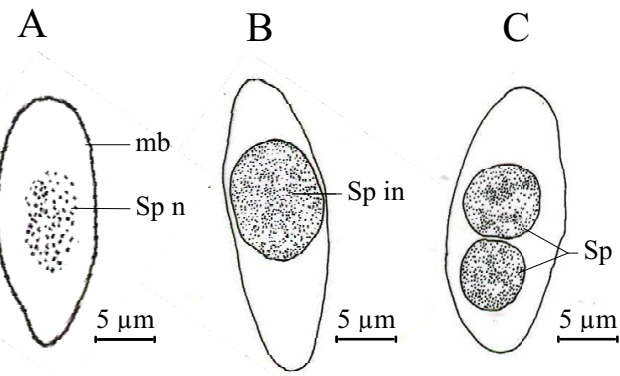

Fig. 6. Structure des oocystes d'l. belli. $\mathrm{mb}$ : membrane plasmique, $\mathrm{Sp} \mathrm{n}$ : sporoblaste non individualisé (A), $S p$ in : sporoblaste individualisé (B), $S p$ : sporocyste (C).

Fig. 6. Structure of the oocysts of $I$. belli. mb: plasma membrane, $\mathrm{Sp} n$ : non individualized sporoblast (A), Sp in: individualized sporoblast (B), Sp: sporocyst (C).

A

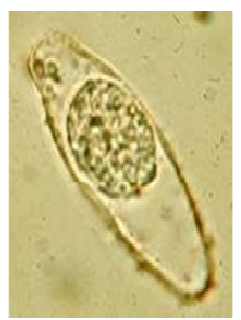

B

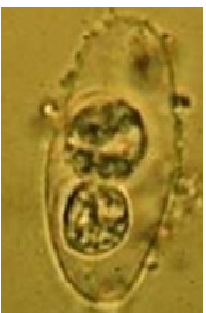

Fig. 7. Structure des oocystes d'I. belli. Après coloration au Lugol : oocyste à sporoblaste individualisé (A) et oocyste à sporocystes (B).

Fig. 7. Structure of the oocysts of $I$. belli. After Lugol coloration: oocyst with individualized sporoblast (A) and oocyst with sporocysts.

non individualisé et à un sporoblaste individualisé est importante alors que les abondances des oocystes à sporocystes ont une variabilité inter saisonnière importante.

\section{DISCUSSION}

Ces observations montrent la présence des oocystes d'l. belli dans le milieu aquatique en zone tropicale et traduisent la contamination fécale des eaux. De même, les résultats des travaux de Kenmogne et al. (2010) ont révélé la présence des formes de résistance des entéropathogènes tels que les kystes d'Entamoeba hystolitica et Giardia sp. avec des abondances faibles qui seraient probablement liées aux techniques des concentrations et d'identification des échantillons. D'ailleurs, ces résultats corroborent ceux obtenus par Ajeagah et al. (2007) révélant la présence des formes de résistance de deux protozoaires entéropathogènes (Cryptosporidium et Giardia). Toutefois, la distribution des 


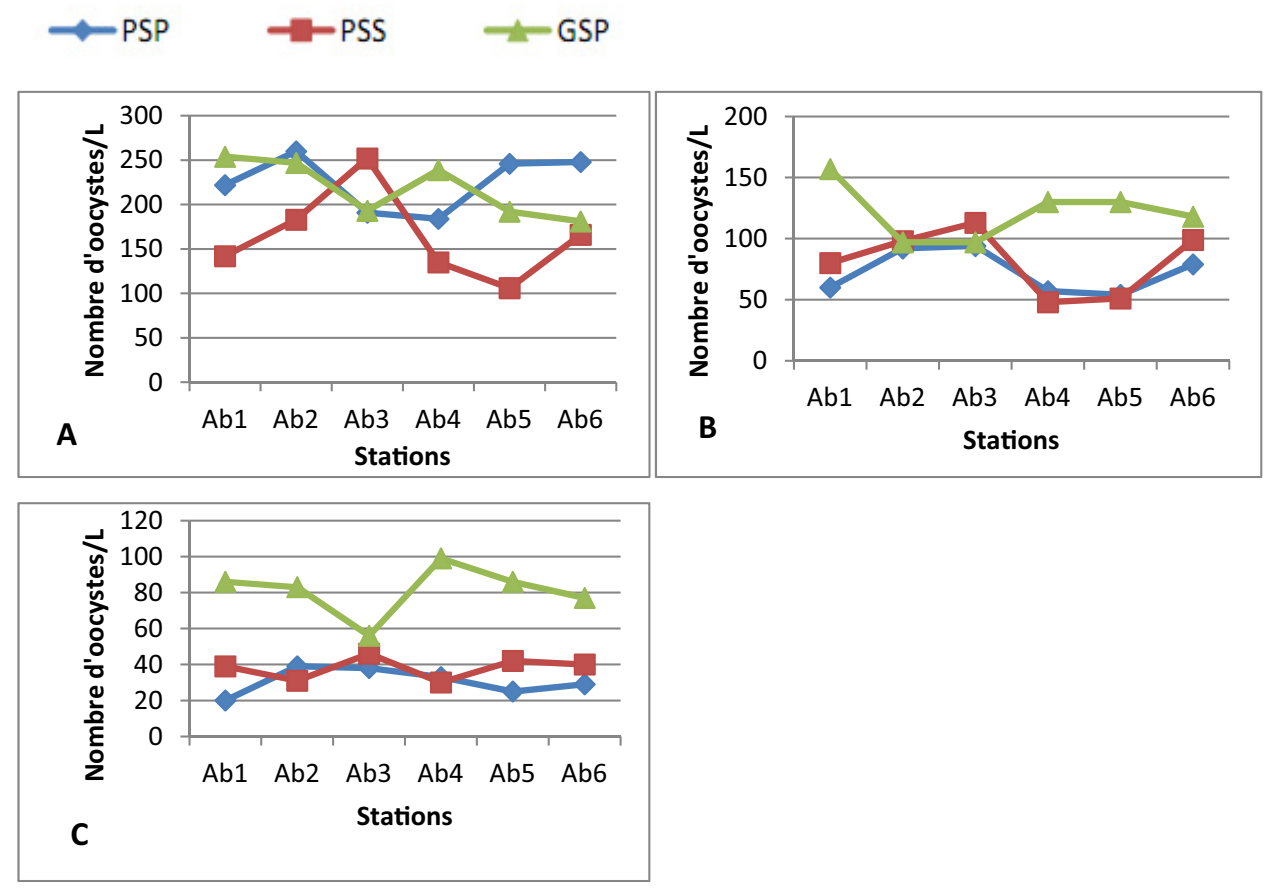

Fig. 8. Variation spatio-temporelle des abondances des oocystes à sporoblaste non-individualisé $(A)$, oocystes à sporoblaste individualisé (B) et oocystes à sporocystes $(\mathrm{C})$ pendant la période d'étude.

Fig. 8. Spatio-temporal variation of the abundance of oocysts with non individualized sporoblast $(A)$, oocyst with individualized sporoblast $(B)$ and oocysts with sporocysts $(C)$ during the study period.

tailles d'oocystes ne présente aucune liaison avec la charge organique pour les petites et moyennes tailles contrairement aux grandes tailles. Ceci résulterait probablement des sérotypes (au niveau de taille) du parasite ingéré par des sujets infectés car en dehors de potentielles mutations, ledit parasite produirait des oocystes de même taille que ceux ingérés par l'hôte en amont. La corrélation significativement positive, obtenue entre la turbidité et les oocystes de grande taille, pourrait s'expliquer par la liaison (électrostatique et Van der Valls) des oocystes aux particules en suspension qui influencerait la turbidité de l'eau. L'Abiergué est une source de dissémination des oocystes d'l. belli dans les quartiers de son bassin versant dépourvus de systèmes d'épuration car Liu et al. (2012) souligne que les processus d'ultrafiltration, de sédimentation et le traitement chimique peuvent réduire l'abondance des oocystes d'l. belli et par conséquent la transmission d'isosporose.

Les observations au microscope des oocystes de ce pathogène à émergence montrent une prédominance des formes non sporulées (sporoblaste nonindividualisé et sporoblaste individualisé) sur les formes sporulées qui 
sont responsables de l'hyperinfection chez l'hôte (Navaneethan et al., 2012). La forte densité des oocystes immatures serait le résultat de la faible oxygénation du plan d'eau. À ce propos, Garcia (2007) et Lindsay et al. (1997) relèvent que la sporulation a lieu dans un milieu oxygéné. Cependant, aucune corrélation significative n'est obtenue entre la teneur en oxygène et les oocystes sporulés. Néanmoins, une corrélation positive et significative $(p=0,05)$ est observée entre les ions nitrates qui sont très mobiles dans l'eau et les oocystes à sporocystes. Or, les nitrates témoignent d'une oxygénation assez bonne mais avec de l'oxygène sous forme liée dans l'eau $\left(\mathrm{NO}_{\mathrm{x}}\right)$ justifiant la corrélation avec les oocystes sporulés. De manière globale, les nitrates et les oocystes à sporocytes sont plus présents en GSP et traduirait le drainage des intrants agricoles et des matières fécales du bassin versant dans l'hydrosystème.

Les faibles densités des oocystes à sporocystes et les fortes densités des oocystes à sporoblaste non individualisé enregistrées au niveau spatio-temporel montrent une sporulation lente. Cette observation ne s'éloigne pas du taux de sporulation de $27 \%$ obtenu par Jongwutives et al. (2007) sur les oocystes excrétés.

Le $\mathrm{pH}$ a présenté une corrélation négativement significative avec les oocystes à sporoblaste non individualisé $(p=0,01)$ ainsi que le $\mathrm{CO}_{2}$ dissous qui a eu une corrélation négativement significative avec les oocystes à sporoblaste individualisé et les oocystes à sporocystes $(p=0,05)$ (Tab. I). À cet effet, la pollution ralentirait le processus de sporulation et par conséquent inhiberait le caractère infectieux des oocystes de l'Abiergué et d'autres milieux aquatiques pollués contrairement aux $\mathrm{pH}$ acides du tube digestif de l'hôte qui accompagnent le cycle biologique du parasite à travers la libération des sporozoïtes.

Bien que dans cette étude la température n'ait aucune corrélation avec les formes des oocystes, ce paramètre aurait un rôle déterminant dans le processus de sporogénèse car ce phénomène se déroule à une température inférieure à $37{ }^{\circ} \mathrm{C}$ après excrétion (Lindsay et al., 1997 ; Garcia, 2007) et également dans un environnement oxygéné. Cependant, le climat étant tropical, les variations de température sont trop faibles pour mettre en évidence un effet sur les oocystes (Tab. I).

Sur le plan temporel, la distribution des oocystes montre une forte abondance durant la PSP et la GSP. Ceci s'expliquerait par la contribution des torrents dans le processus d'apport d'oocystes contenu dans le péril fécal et les décharges domestiques dans l'hydrosystème lors des pluies. Pendant la saison des pluies, de grandes quantités d'eau sont drainées des pentes du bassin versant jusqu'au cours d'eau lessivant les matières organiques et en plus, les riverains profitent des pluies pour libérer les contenus des latrines à fosse perdue dans l'hydrosystème. Cela étant, les oocystes sont également observés durant la PSS avec des abondances élevées, semblables à celles de la PSP et de la GSP. 


\section{CONCLUSION}

Cette étude a permis l'isolement et la mise en évidence des oocystes d'l. belli dans l'Abiergué. Les analyses physico-chimiques et biologiques révèlent une pollution organique du cours d'eau et une contamination de l'amont à l'aval. II est à noter une influence des paramètres physico-chimiques sur l'excystation et la viabilité de cet entéropathogène. Cependant, les oocystes non sporulés (oocystes à sporoblaste non individualisé et ceux à sporoblaste individualisé) sont les plus abondants dans le milieu aquatique aussi bien au niveau des stations qu'au niveau des saisons bien que la GSP ait enregistré les plus fortes abondances. La prédominance des oocystes non sporulés prouve l'existence d'une contamination et exprime un risque d'exposition pour les populations même si les formes sporulées les plus dangereuses sont moins représentées. Par ailleurs, la présence d'une gamme variée de tailles d'oocystes du parasite est importante pour le choix des filtres d'un éventuel système de traitement. Pour compléter cette étude, il est envisageable d'étudier les relations entre la qualité de l'eau et le caractère infectieux des oocystes et faire un rapport entre la morphométrie des oocystes et la susceptibilité de l'hôte à l'isosporose.

\section{RÉFÉRENCES BIBLIOGRAPHIQUES}

Ajeagah G., Njine T., Nola M., Foto S. \& Wouafo M., 2007. Measuring resistant forms of two pathogenic protozoa (Giardia spp. and Cryptosporidium spp.) in two aquatic biotopes in Yaoundé (Cameroon). Cahier d'études et de recherches francophones/santé 17 (3) : 72-167.

Ajeagah G.A., Njine T., Bilong B.C.F., Foto S.M., Wouafo N.M., Nola M., Di Giovanni G.D. \& Huw S., 2010. Seasonal distribution of enteric opportunistic Cryptosporidium Spp. oocysts and Giardia Spp. cysts in a tropical water basin, Cameroon. Water 2 : 44-57.

Ajeagah G., Bikibe J.F. \& Longo F., 2013. Qualité bioécologique d'un milieu lacustre hyper-eutrophisé en zone équatoriale (Afrique Centrale) : peuplement de protozoaires ciliés et macro invertébrés bentho-aquatiques. Afrique Science 09 (2) : 50-66.

Angelier E., 2000. Écologie des eaux courantes. Edition Tec \& Doc, Paris, 199 p.

Cranenbonk R.J., Kodde C.J., Chipeta D., Zijlstra E.E. \& Sluiters J.F., 2003. Cryptosporidium parvum and Isospora belli infections among patients with and without diarrhea. East African Medicine Journal 80 : 398-401.

Garcia L.S., 2007. Intestinal protozoa (Coccidia and Microsporidia) and algae. Diagnostic Medical Parasitology. 5th edition, ASM Press. Washington DC, 57-101.

Gomez A.H.S., Pacheco M.A.S.R., Fonseca Y.S.K., Cesar N.P.A., Dias H.G.G. \& Silva R.P., 2002. Detection of Cryptosporidium $\mathrm{sp}$. in water from natural springs. Institut Adolfo Lutz Review 61 : 59-69.

INC, 2008. Carte topographique de la région de Yaoundé au 1/10000. Yaoundé: Institut National de Cartographie, 4 feuilles.

Jongwutiwes S., Putaporntip C., Charoenkorn M., Iwasaki T. \& Endo T., 2007. Morphologic and molecular characterization of Isospora belli oocysts from patients in Thailand. American Journal of Tropical Medicine and Hygiene 77 : 107-112. 
Kenmogne K.R.G., Rosillon F., Mpakam G.H. \& Nono A., 2010. Enjeux sanitaires, socio-économiques et environnementaux liés à la réutilisation des eaux usées dans le maraîchage urbain : cas du bassin versant de l'Abiergué (YaoundéCameroun). VertigO-la revue électronique en sciences de l'environnement $10(2): 1-23$.

Levêque C., 2001. Écologie : de l'écosystème à la biosphère. Paris, Dunod, $502 \mathrm{p}$

Lindsay D.S., Dubey J.P. \& Blagburn B.L., 1997. Biology of Isospora ssp from humans, nonhuman primates and domestic animals. Clinical Microbiology Review 10 : 19-34.

Lindsay D.S., Upon S.J. \& Weiss L.M., 2007. Isospora, Cyclospora and Sarcocystis. In Manual of Clinical Microbiology, Murray et al. (Eds.), 9th ASM Press, Washington, DC, 2113-2121.

Liu P., Hill V.R., Hahn D., Johnson T.B., Pan Y., Jothikumar N. \& Moe C.L., 2012. Hollow-fiber ultrafiltration for simultaneous recovery of viruses, bacteria and parasites from reclaimed water. Journal of Microbiology Methods 88 (1) : 61-155.

Meamar A.R., Rezaian M., Zare-Mirzaei A., Zahabium F., Faghihi A.H., Oormazdi H. \& Kia E.B., 2009. Severe diarrhea due to Isospora belli in a patient with thymoma. Journal of Microbiology Immunology and Infection 42 : 526-529.

Mircean V., GyörkeA. \& Cozma V., 2012. Veterinary Parasitology 184 (2-4) : 9-325.

Navaneethan U., Venkatesh P.G., DownsKelly E. \& Shen B., 2012. Isospora belli superinfection in a patient with eosinophilic gastroenteritis-A diagnostic challenge. Journal Crohns Colitis 6 (2) : 9-236.

Ndam Ngoupayou. J.R., Apouamoun R., Youego S.J.R., Ngnike P.M., Boeglin
J.-L. \& Bedimo J.P., 2007. Transfert d'eau et de matières dans un écosystème forestier urbanisé en Afrique : le bassin versant de la Mefou au Sud du Cameroun. Actes des JSIRAUF, Hanoi, 1-6.

ONU, 2006. L'eau, une responsabilité partagée. $2^{\mathrm{e}}$ Rapport des Nations-Unis sur la mise en valeur des ressources en eau, $45 \mathrm{p}$.

Ousmane O.C., 2010. Diagnostic de l'isosporose chez les patients VIH diarrhéiques à Bamako (Mali). Thèse, Université de Bamako, $82 \mathrm{p}$.

Pedro M.L.G \& Germano M.I.S., 2001. Water: A national security problem. Food Hygiene Review 15 : 15-18.

Rodier J., 2009. L'analyse de l'eau. $9^{e}$ édition, Dunod, Paris, 1526 p.

Santoir C., 1995. La pédologie. Atlas régional Sud Cameroun. Inc. Santoir C. \& Bopda A. (Eds.), Cameroun: ORSTOM et MINREST, $53 \mathrm{p}$.

Sasaki M., Tanaka A., Nishimura T., Tsujikawa T., Andoh A. \& Ishizuka I., 2004. A case of malabsorption syndrome caused by isosporiasis in an immunocompetent patient. Journal Gastroenterological 39 : 9-88.

Sonia F.O.S., Hugo D.S., Edson S.S.J., Carlos E.A., Elisângela P.S.-L., Cesar A.S.T.V.-C. \& Marco T.A.G., 2010. Environment monitoring of opportunistic protozoa in rivers and lakes in the neotropics based on yearly monitoring. Water Quality Exposition Health 2 : 97104.

Suchel B., 1987. Les climats du Cameroun. Thèse de Doctorat d'État. Université de Bordeaux III, $1186 \mathrm{p}$.

Udeh E.O., Goselle O.N., D-Popova D.D., Abelau M., Popov T.V., Jean N. \& David J.S., 2008. The prevalence of intestinal protozoans in HIV/AIDS patients in Abuja, Nigeria. Science World Journal 3 (3) : 1-4. 
Verdier R.I., Fitzgerald D.W., Johnson W.D. Jr \& Pape J.W., 2000. Trimethoprim-sulfamethoxazole compared with ciprofloxacin for treatment and prophylaxis of Isospora belli and Cyclospora cayetanensis infection in HIV-infected patients. A randomized controlled trial. Annual International of Medicine, 4-885.

Villeneuve V., Légaré S., Painchaud J. \& Vincent W., 2006 Dynamique et modélisation de l'oxygène dissous en Rivière.
Revue des sciences de l'eau 19(4) : 259-274.

Yongsi N.B., Salem G. \& Bruneau J.C. 2008. Epidémiologie géographique des maladies diarrhéiques à Yaoundé (Cameroun). M@ppemonde 1 : 1-17.

Yongué-Fouateu R., 1986. Contribution à l'étude pétrographique de l'altération et des faciès de curassement des gneiss migmatiques de la région de Yaoundé. Thèse de Doctorat du $3^{e}$ cycle. Université de Yaoundé, $214 \mathrm{p}$. 